\title{
Hairpin DNA-functionalized gold nanorods for mRNA detection in homogenous solution
}

\author{
Guoke Wei \\ Jun $\mathrm{Yu}$ \\ Jinliang Wang \\ Peng Gu \\ David J. S. Birch \\ Yu Chen
}




\title{
Hairpin DNA-functionalized gold nanorods for mRNA detection in homogenous solution
}

\author{
Guoke Wei, ${ }^{a, b}$ Jun Yu, ${ }^{c}$ Jinliang Wang, ${ }^{b}$ Peng Gu, ${ }^{a}$ David J. S. Birch, ${ }^{a}$ and Yu Chen ${ }^{a, *}$ \\ aUniversity of Strathclyde, Photophysics Group, Center for Molecular Nanometrology, Department of Physics, SUPA, John Anderson Building, \\ 107 Rottenrow, Glasgow G4 0NG, United Kingdom \\ ${ }^{\mathrm{b} B e i h a n g}$ University, Department of Physics, 37 Xuequan Road, Haidan, Beijing 100191, China \\ 'University of Strathclyde, Strathclyde Institute of Pharmacy and Biomedical Sciences, Royal College, 204 George Street, Glasgow G1 1XW, \\ United Kingdom
}

\begin{abstract}
We report a fluorescent probe for mRNA detection. It consists of a gold nanorod (GNR) functionalized with fluorophore-labeled hairpin oligonucleotides (hpDNA) that are complementary to the mRNA of a target gene. This nanoprobe was found to be sensitive to a complementary oligonucleotide, as indicated by significant changes in both fluorescence intensity and lifetime. The influence of the surface density of hpDNA on the performance of this nanoprobe was investigated, suggesting that high hybridization efficiency could be achieved at a relatively low surface loading density of hpDNA. However, steady-state fluorescence spectroscopy revealed better overall performance, in terms of sensitivity and detection range, for nanoprobes with higher hairpin coverage. Time-resolved fluorescence lifetime spectroscopy revealed significant lifetime changes of the fluorophore upon hybridization of hpDNA with targets, providing further insight on the hybridization kinetics of the probe as well as the quenching efficiency of GNRs. ๑ 2016 Society of Photo-Optical Instrumentation Engineers (SPIE) [DOI: 10.1117/1.JBO.21.9 097001]
\end{abstract}

Keywords: gold nanorods; hairpin DNA; mRNA detections; fluorescence lifetime.

Paper 160218RR received Apr. 6, 2016; accepted for publication Aug. 16, 2016; published online Sep. 8, 2016.

\section{Introduction}

Messenger RNA (mRNA) plays a key role in the cellular production of protein. Detection of mRNA biomarkers with good specificity and sensitivity will enable an early stage diagnosis of diseases such as cancer and assist in monitoring and evaluating the efficacy of treatment. Moreover, detection of mRNA provides valuable information for understanding the fundamental metabolism of cells. ${ }^{1,2}$ For this purpose, a number of techniques have been developed. ${ }^{3}$ Among them, nucleic acids-based detection and quantification methods have attracted substantial interest since nucleic acids possess the inherent property to selectively bind to the complementary targets through WatsonCrick base-pairing. One of the promising approaches to detect mRNA, often denoted as molecular beacon (MB), is a hairpinshaped oligonucleotide with a fluorophore-quencher pair that undergoes a spontaneous fluorogenic conformational change upon hybridization with the complementary nucleic acid target. ${ }^{4,5}$ It offers great opportunities in homogeneous assay of mRNA and also the capability of real-time monitoring of the expression of mRNAs in living cells, even down to the single-cell level, resulting from its high sensitivity and enhanced specificity. ${ }^{6-9}$

However, the traditional MB suffers from problems of lacking universal organic quenchers ${ }^{10}$ and requiring transfection reagents for cellular internalization. ${ }^{8}$ Recent studies show that these limitations can be elegantly addressed by gold nanoparticles (AuNP). It has been proven that AuNP are highly efficient quenchers for a range of organic fluorophore ${ }^{10-13}$ and exhibit long-range fluorescence quenching capability. ${ }^{14}$ Moreover, $\mathrm{Au}$ nanospheres (AuNS) functionalized by oligonucleotides display several fascinating features, including highly efficient cellular uptake without the need of transfection reagents, extraordinary intracellular stability against enzymatic degradation, and enhanced binding capability of complementary nucleic acids. ${ }^{15-17}$ Additionally, AuNP are biocompatible and have versatile surface modifications, especially through the well-established gold-thiol chemistry. By taking the advantages of AuNP and $\mathrm{MB}$, a nanoprobe has been recently developed, in which AuNS were covalently functionalized by hairpin oligonucleotides dually labeled with fluorophore and thiol. ${ }^{18}$ This nanoprobe shows promising applications in simultaneous multianalysis of nucleic acid with high sensitivity and specificity. ${ }^{18-20}$ More importantly, spatial-temporal information about nucleic acid targets in living cells can be acquired by using this AuNP-MB conjugate as an intracellular probe since the fluorophores are still anchored to the AuNP rather than being released into the cytoplasm when binding to the targets. ${ }^{21,22}$

Compared to AuNS, gold nanorods (GNRs) exhibit excellent shape-dependent optical properties. By varying the aspect ratio, the longitudinal plasmon band of GNR can be finely tuned from visible to near-infrared regions. ${ }^{23,24}$ This is of particular interest for biological applications due to the high transmission of tissues in the near-infrared window $(650$ to $900 \mathrm{~nm}){ }^{25}$ In addition to the large absorption and scattering cross section, GNRs have strong two-photon luminescence arising from the localized surface plasmon resonance. ${ }^{26-28}$ Two-photon excitation holds promise for intracellular studies as it has higher spatial resolution, deeper penetration, and less photo-damage compared to single-photon excitation. Interestingly, two-photon 


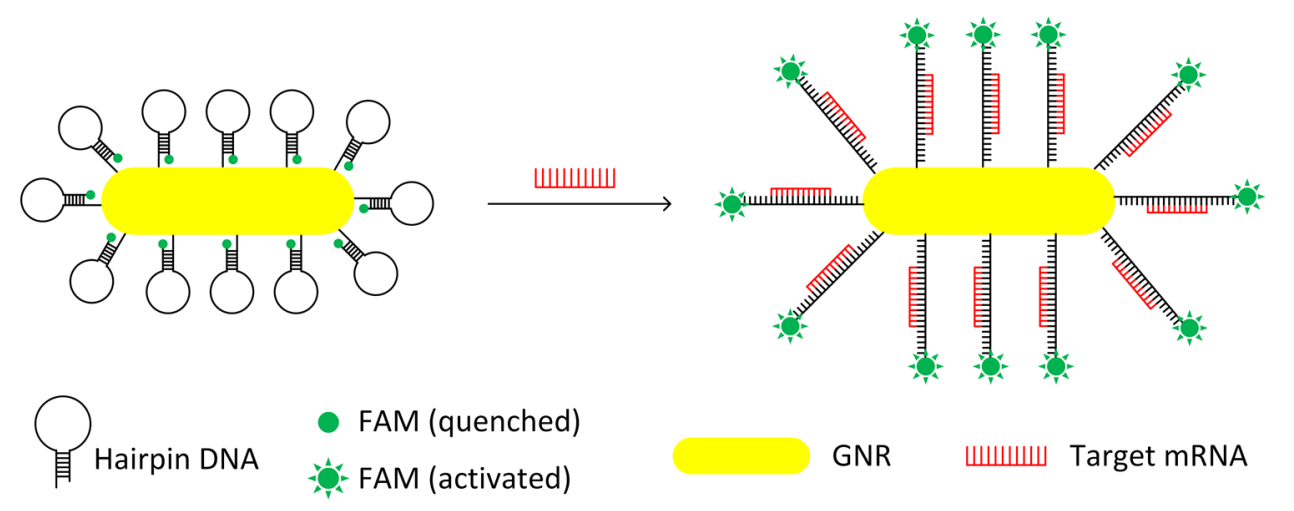

Fig. 1 Schematic illustration of hairpin DNA-functionalized GNR for mRNA detection.

luminescence from GNR shows a characteristic short lifetime $(<100 \mathrm{ps})$ distinguishable from many organic dyes and autofluorescence, offering benefit in fluorescence lifetime imaging microscopy (FLIM). ${ }^{29}$ These unique features make GNR promising candidates for numerous biological and biomedical applications, including biological imaging, ${ }^{30}$ gene/drug delivery, ${ }^{31}$ and photothermal therapy. ${ }^{32}$

Considering the unique properties of GNR, it is expected that GNR coupled with hairpin oligonucleotides will offer great opportunity in mRNA detection and imaging. Recently, we have reported an RNA nanoprobe based on functionalized GNR and the influence of hairpin structure on the quenching efficiency of the energy transfer pair of GNR and Cy5. ${ }^{33}$ Due to the steric structure of hpDNA and difficulty in completely replacing the CTAB bilayer surrounding GNRs with biomolecules, the functionalization of GNRs with hpDNA has been found challenging and has been less reported. Here, to the best of our knowledge, we report for the first time the functionalization of GNR with 6-carboxyfluorescein (FAM)-labeled hairpin DNA (hpDNA) (Fig. 1), and influence of the synthesis condition on the performance of this nanoprobe in target mRNA detection using both steady-state and time-resolved fluorescence spectroscopies. FAM was chosen as it can be attached to the 3 ' end and allows reliable attachment of oligonucleotide with thiol molecule modified in the 5' end. Our results show that the GNR-hpDNA conjugates are highly sensitive probes for mRNA detection with high signal-to-background ratio. Moreover, we investigated the influence of the surface density of hpDNA on GNR on the performance of this nanoprobe and found that high hybridization efficiency could be achieved at a relatively low surface loading density of hpDNA. The fluorescence lifetime measurements revealed the recovery of fluorescence lifetime in the hybridization events, indicating the conformational change of hpDNA when binding to target mRNA complement. Significantly, fluorescence lifetime spectroscopy is demonstrated as a powerful tool for fluorescencebased mRNA detection.

\section{Experimental Section}

\subsection{Materials}

All chemicals were purchased from Sigma-Aldrich and used as received. All buffers were prepared using nuclease-free water obtained from Sigma-Aldrich. Thiolated oligonucleotides and the corresponding complementary oligonucleotides were purchased from Eurofins MWG Operon and Integrated DNA Technologies, respectively.

\subsection{Synthesis of Gold Nanorods}

GNRs were synthesized according to the silver-assisted seedmediated growth method. ${ }^{34,35}$ Briefly, $2.5 \mathrm{~mL}$ of $0.001 \mathrm{M}$ $\mathrm{HAuCl}_{4}$ was mixed with $7.5 \mathrm{~mL}$ of $0.2 \mathrm{M}$ hexadecyltrimethylammonium bromid (CTAB) solution. Next, $0.6 \mathrm{~mL}$ of freshly prepared ice-cold $0.01 \mathrm{M} \mathrm{NaBH}_{4}$ was quickly added to the solution under vigorous stirring, forming a brownish-yellow seed solution. The seed solution was vigorously stirred for another $2 \mathrm{~min}$ and then kept undisturbed at room temperature for $3 \mathrm{~h}$ before use. To make a growth solution, $200 \mathrm{~mL}$ of $0.2 \mathrm{M}$ CTAB solution was gently mixed with the following solutions in the following order: $200 \mathrm{~mL}$ of $0.001 \mathrm{M} \mathrm{HauCl}_{4}, 8 \mathrm{~mL}$ of $0.004 \mathrm{M} \mathrm{AgNO}_{3}, 2.8 \mathrm{~mL}$ of $0.0778 \mathrm{M}$ ascorbic acid. Then $0.4 \mathrm{~mL}$ of the colloidal gold seeds were added to the growth solution and the reaction mixture was left on the bench undisturbed overnight. The obtained nanorods were spun down by centrifugation $(14,500 \mathrm{rpm}, 12 \mathrm{~min})$ and finally resuspended in $2 \mathrm{~mL}$ of distilled water. This process produced GNRs of diameter $12.7 \pm 1.8 \mathrm{~nm}$ and length $51.6 \pm 8.2 \mathrm{~nm}$ as derived from TEM analysis [Fig. 2(a)], and a longitudinal surface plasmon resonance (LSPR) peak centered at $800 \mathrm{~nm}$. Experimentally, reproducibility of further functionalization was ensured by producing nanorods of similar surface plasmon resonance property.

\subsection{Ligand Exchange of Nanorods}

The CTAB surfactant on the GNR surface was replaced with mercaptohexanoic acid (MHA) using a round-trip phase transfer ligand exchange approach. ${ }^{36}$ First, the CTAB-coated GNRs (NR-CTAB) were extracted from the aqueous phase to the organic phase by dodecanethiol (DDT) upon the addition of acetone following a few second swirling. During this process, the CTAB was displaced by DDT, resulting in DDT-coated GNR (NR-DDT). The volume ratio of the concentrated NRCTAB solution, DDT, and acetone was 1:1:4. The excess DDT was then diluted by adding an aliquot of toluene and five aliquots of methanol and washed away by centrifugation (5000 rpm, $8 \mathrm{~min}$ ). The NR-DDT was resuspended in $1 \mathrm{~mL}$ toluene by brief sonication. Next, the GNRs were extracted back to the aqueous phase using MHA as the exchanged ligand. The NR-DDT was added to $9 \mathrm{~mL}$ of $0.01 \mathrm{M}$ MHA in toluene at 

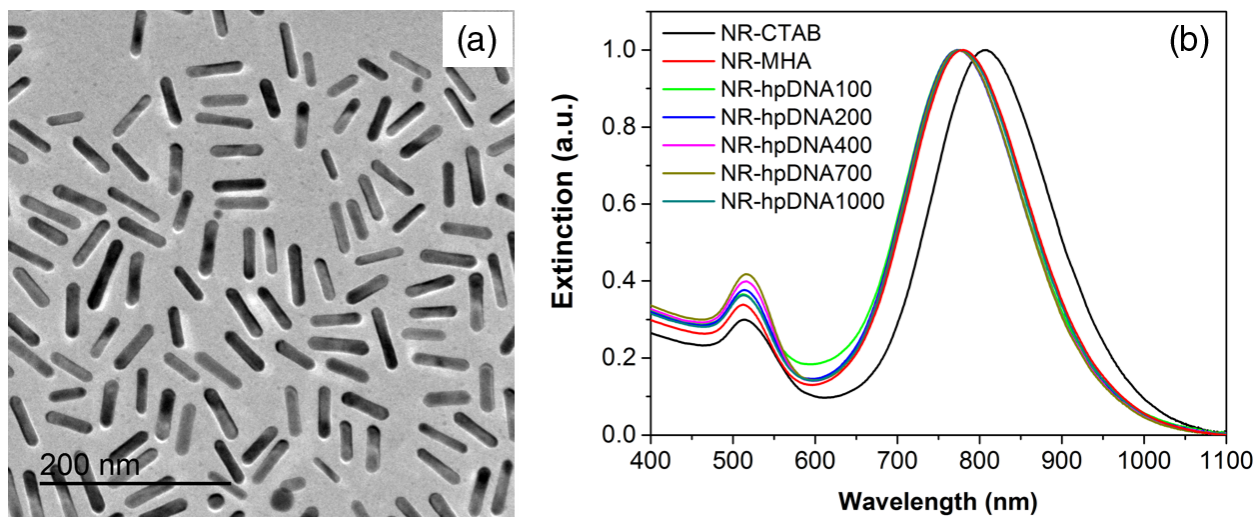

Fig. 2 (a) TEM image of the GNR. The scale bar is $200 \mathrm{~nm}$. (b) Extinction spectra of GNR made with CTAB and GNRs with surface modifications of MHA and hpDNA-FAM. The NR-CTAB was suspended in distilled water, whereas NR-MHA and NR-hpDNA-FAM were suspended in $1 \times \mathrm{TBE}(\mathrm{pH} 8.3)$ and $10 \mathrm{mM}$ phosphate buffer ( $\mathrm{pH} 7.5)$, respectively.

$\sim 90^{\circ} \mathrm{C}$ and vigorously stirred. Reflux and stirring continued until visible aggregation was observed (within $\sim 15 \mathrm{~min}$ ), indicating that MHA had replaced the DDT. The MHA-coated GNR (NR-MHA) were then left to sediment, washed twice with aliquots of toluene via decantation, and once with an aliquot of isopropanol to remove all reaction byproducts and excess MHA. Finally, the NR-MHA was resuspended in $1 \times$ Trisborate-EDTA (TBE) buffer (pH 8.3) with a high concentration of $\sim 100 \mathrm{nM}$. The GNR concentrations were determined by optical absorption using the reported extinction coefficients. ${ }^{37}$

\subsection{Hairpin DNA Functionalization of Nanorods}

A thiolated hairpin DNA (hpDNA) with a 6-carboxyfluorescein (FAM) label in the 3' end [5'-HS- $\left(\mathrm{CH}_{2}\right)_{6}$-TTTTT GCGAG TTG GTG AAG CTA ACG TTG AGG CTCGC-FAM-3'; the italic bases represent the stem sequence] was designed to recognize a 21-nucleotide region of c-myc mRNA. A 5-base polythymine spacer was inserted following the 5' thiol in order to reduce selfadsorption of DNA to the surface of GNR. ${ }^{38,39}$ The disulfide bonds of thiolated hpDNA were reduced by tris(2-carboxyethyl)phosphine hydrochloride (TCEP) in $1 \times$ TE buffer $(\mathrm{pH}$ 8.0) with TCEP/DNA molar ratio of 100:1. After 60-min incubation at room temperature while shaking, the activated DNA was precipitated from the mixture by sodium acetate and ethanol. Specifically, to the reduced DNA solution, appropriate quantities of $3 \mathrm{M}$ sodium acetate and $100 \%$ ethanol were added so that the final salt concentration was $0.3 \mathrm{M}$ and the final ethanol concentration was $70 \%$. The mixture was incubated $20 \mathrm{~min}$ at $-20^{\circ} \mathrm{C}$ and then spun for $5 \mathrm{~min}$ at $13,000 \mathrm{rpm}$. The supernatant was discarded and the pellet was resuspended in $1 \times$ TE buffer ( $\mathrm{pH} 8.0)$.

The NR-MHA was conjugated with hpDNA by a salt aging process. ${ }^{40,41}$ The hpDNA of different concentrations (5.0, 3.5, 2.0, 1.0, $0.5 \mu \mathrm{M})$ were incubated with $5 \mathrm{nM}$ NR-MHA, respectively, in $10 \mathrm{mM}$ phosphate buffer (pH 7.0) with 0.02 (wt./vol.) $\%$ SDS at room temperature. After 3-h incubation, $10 \mu \mathrm{L}$ of salting solution containing $500 \mathrm{mM} \mathrm{NaCl}$ and $0.02 \%$ sodium dodecyl sulfate (SDS) in $10 \mathrm{mM}$ phosphate buffer $(\mathrm{pH} 7.0)$ was added to the mixture every $60 \mathrm{~min}$. This step was repeated for a total of five times to reach a final $\mathrm{NaCl}$ concentration of $100 \mathrm{mM}$. The salted sample was further incubated at room temperature for $16 \mathrm{~h}$. The NR-hpDNA conjugates were purified of excess reagents via centrifugation at 13,000 rpm for $15 \mathrm{~min}$ at $4^{\circ} \mathrm{C}$. The precipitate was washed four times with washing buffer (10 $\mathrm{mM}$ phosphate buffer $+0.02 \%$ SDS, $\mathrm{pH} 7.5)$, and an additional three times with $10 \mathrm{mM}$ phosphate buffer $(\mathrm{pH} 7.5)$ by repetitive centrifugation and dispersion, which was finally resuspended in $10 \mathrm{mM}$ phosphate buffer $(\mathrm{pH} 7.5)$ and stored at $4^{\circ} \mathrm{C}$.

\subsection{Quantitation of Hairpin DNA Loading on Nanorods}

The hpDNA loaded on GNR was quantified by chemical displacement and fluorescence spectroscopy. ${ }^{38}$ The purified NRhpDNA conjugates were incubated in $20 \mathrm{mM}$ mercaptoethanol (ME) overnight with shaking at room temperature, displacing the hpDNA from GNR. The released hpDNAs were then separated from GNR via centrifugation $(13,500 \mathrm{rpm}, 15 \mathrm{~min})$. The fluorescence of the displaced hpDNA was measured and converted to molar concentration of hpDNA by interpolation from a standard linear calibration curve, which was prepared with known concentrations of fluorophore-labeled hpDNA with identical buffer $\mathrm{pH}$, ionic strength, and ME concentration. The average number of hpDNA per GNR was obtained by dividing the molar concentration of hpDNA by the original GNR concentration.

\subsection{Hybridization Efficiency of NR-hpDNA probes}

Hybridization efficiency was quantified according to the published protocol. ${ }^{38}$ TAMRA-labeled complementary DNA (TMR-c-DNA) was incubated with NR-hpDNA under hybridization conditions ( $3 \mu \mathrm{M}$ TMR-c-DNA, $10 \mathrm{mM}$ phosphate buffer with $100 \mathrm{mM} \mathrm{NaCl}, \mathrm{pH}$ 7.5, 24 h). Nonhybridized c-DNA-TMR was removed and rinsed three times by $10 \mathrm{mM}$ phosphate buffer (pH 7.5) through centrifugation $(13,500 \mathrm{rpm}, 15 \mathrm{~min})$. After that, the TMR-c-DNAs were dehybridized by addition of $\mathrm{NaOH}$ (final concentration $50 \mathrm{mM}$, pH 11 to 12, 2 h). The dehybridized TMR-c-DNAs were then separated from the mixture by centrifugation, and neutralized by addition of $1 \mathrm{M} \mathrm{HCl}$. The concentration of dehybridized TMR-c-DNA and the corresponding hybridization efficiency were determined by fluorescence spectroscopy analysis. 


\subsection{Hybridization Kinetics}

The c-DNA was used to investigate the hybridization kinetics of the nanoprobes. The hybridization experiments were carried out in the hybridization buffer $(10 \mathrm{mM}$ phosphate buffer of $\mathrm{pH} 7.5$, $100 \mathrm{mM} \mathrm{NaCl}$ ) containing $0.22 \mathrm{nM}$ nanoprobes and $880 \mathrm{nM} \mathrm{c}$ DNA. The excitation and emission wavelengths were 490 and $517 \mathrm{~nm}$ for fluorescein, respectively.

\subsection{Sensitivity Experiment}

The nanoprobes $(0.22 \mathrm{nM})$ were incubated with varying concentrations of c-DNA $(0,1,5,10,20,30,40,50,80,100,200$, and $300 \mathrm{nM}$ ) in the hybridization buffer for $2 \mathrm{~h}$ at $37^{\circ} \mathrm{C}$ before measuring the fluorescence recovery.

\subsection{Fluorescence Lifetime Measurements}

Time-resolved fluorescence measurements were performed using the time-correlated single-photon counting technique on an IBH Fluorocube fluorescence lifetime system (Horiba Jobin Yvon IBH Ltd., Glasgow, UK) equipped with both excitation and emission monochromators. A pulsed light-emitting diode of $474 \mathrm{~nm}$ operating at $1 \mathrm{MHz}$ repetition rate was used as the excitation source. A longpass filter of $505 \mathrm{~nm}$ was used to minimize the detection of excitation light. Fluorescence decays were measured at the magic angle $(54.7 \mathrm{deg})$ to eliminate polarization artifacts. Data analysis was performed using nonlinear least squares with the IBH iterative reconvolution software (DAS6 data analysis package). The fluorescence intensity decays were analyzed in terms of the multiexponential model as the sum of individual single exponential decays

$I(t)=\sum_{i} \alpha_{i} \exp \left(-\frac{t}{\tau_{i}}\right)$,

where $\tau_{i}$ are the decay times and $\alpha_{i}$ is the associated amplitudes. The fractional contribution of each lifetime component to the steady-state intensity is represented by

$f_{i}=\alpha_{i} \tau_{i} / \sum_{k} \alpha_{k} \tau_{k}$

The average lifetime $(\bar{\tau})$ is calculated as

$\bar{\tau}=\sum_{i} f_{i} \tau_{i}$

As noted, a very short lifetime component ( $<100$ ps) was found in both cases before and after hybridization. This lifetime is below the system response time limit and is attributed to the scattering of GNR. This was excluded in the multiexponential fittings by deliberately fixing one of the lifetime components at a value of 0.5 channels.

To retrieve the lifetime distributions, a model-free maximum entropy method (MEM) was used, using the software Pulse 5 (MaxEnt Ltd, Cambridge, UK) ${ }^{42}$ It provides a unique solution to fluorescence lifetime data using a broad window of decay terms fit by simultaneous minimization of the $\chi^{2}$ and maximization of a statistical entropy function. The lifetime distribution $h(\tau)$ is related to the fluorescence intensity decay $I(t)$ by

$I(t)=\int_{0}^{\infty} h(\tau) \exp \left(-\frac{t}{\tau}\right) \mathrm{d} \tau$.

\section{Results and Discussion}

The bilayer CTAB on the surface of as-made GNR may not only cause a cytotoxic effect to living cells, ${ }^{43,44}$ but also can be problematic for further surface modification with bioconjugates. ${ }^{23}$ Thus, the CTAB layers were replaced with MHA prior to conjugation with hpDNA, using a round-trip phase transfer ligand exchange approach. ${ }^{36}$ As shown in Fig. 2(b), the LSPR of the CTAB-coated GNR was centered at $807 \mathrm{~nm}$. This LSPR band was blue-shifted to $780 \mathrm{~nm}$ without significant broadening after the ligand exchange process, indicating a successful ligand exchange without apparent aggregation. The hpDNA was conjugated with GNR via a salt-aging process, ${ }^{40,41}$ in which different molar ratios of hpDNA to GNR, namely 100:1, 200:1, 400:1, 700:1, and 1000:1, were used. The UV-vis spectra showed that the LSPR bands of all hpDNA-functionalized GNRs did not exhibit significant change compared to that of MHA modified GNR (NR-MHA), regardless of different molar ratios of hpDNA to GNR used in the synthesis process [Fig. 2(b)]. This was possibly due to centrifugation processes where a fraction of GNRs with high aspect ratios were inevitably left in the supernatant after each round of centrifugation as the sedimentation velocity was dictated by the hydrodynamic behavior of nanoparticles, ${ }^{45}$ making it difficult to observe the slight changes of LSPR peaks among GNR-MHA with different hpDNA coverages experimentally.

To quantify the average number of hpDNA assembled on a GNR, hpDNA on GNRs were released by ME and the concentration of hpDNA was determined by fluorescence intensity against a standard correlation curve between fluorescence intensity and hpDNA-FAM concentration. ${ }^{38}$ The surface packing density of hpDNA on single GNR was obtained with known GNR particle density. As depicted in Fig. 3, the surface loading of hpDNA on GNR varies with the molar ratio of hpDNA to GNR in the mixture. It is interesting to note that the surface loading of hpDNA reached a maximum of $\sim 114$ at a molar ratio of $400: 1$, and maintained at this value even with higher molar ratios. This is well below the value of maximum loading, $\sim 168$ oligonucleotides per GNR, as predicted by Hill's model. ${ }^{46}$ In this successive study, samples made from molar ratios of $100: 1,200: 1$, and 400:1 were investigated, denoted as NRhpDNA100, NR-hpDNA200, and NR-hpDNA400, respectively. The footprints of hpDNA loading on GNR were calculated to be approximately $114.4,42.9$, and $18.1 \mathrm{~nm}^{2}$ for NR-hpDNA100, NR-hpDNA200, and NR-hpDNA400, respectively.

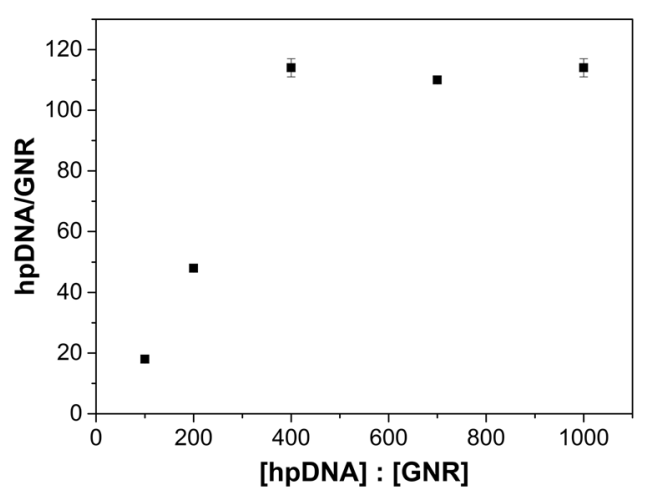

Fig. 3 A correlation between surface loading of hpDNA on each GNR and molar ratio of hpDNA to GNR in the synthesis process. Error bars are one standard deviation from three measurements. 


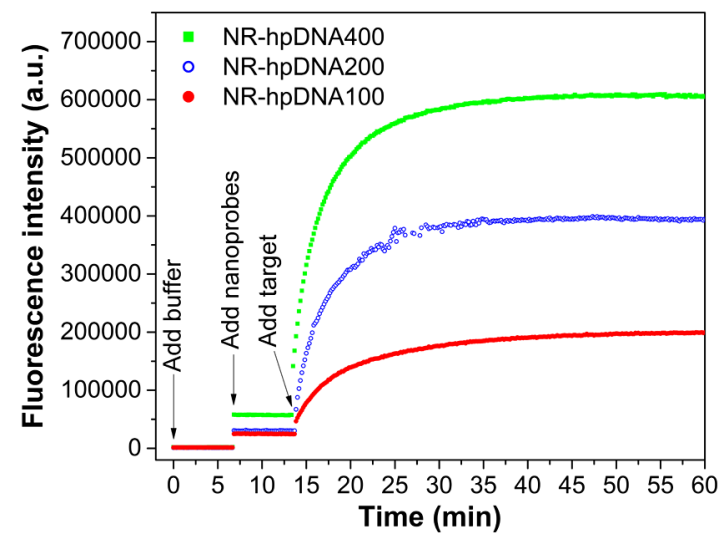

Fig. 4 Kinetic fluorescence measurements of the nanoprobes upon hybridization. The concentrations of NR-hpDNA and c-DNA were 0.22 and $880 \mathrm{nM}$, respectively. Excitation wavelength: $490 \mathrm{~nm}$; fluorescence wavelength: $517 \mathrm{~nm}$.

To test the performance of the nanoprobes, we first examined their fluorogenic responses to the addition of targets. The nanoprobes were exposed to an excess amount of perfectly matched cDNA $(880 \mathrm{nM})$. As demonstrated by the kinetic measurements in Fig. 4, all of the nanoprobes showed an instant fluorescence recovery upon adding c-DNA, and the fluorescence intensities reached saturation levels in short time periods. This is consistent with the previous studies using MB and AuNS-MB conjugates, ${ }^{5,18,47}$ indicating that the GNR-based nanoprobes retained the advantage of MB. In addition, it is noted that the surface packing density of hpDNA had a great impact on the hybridization kinetics of nanoprobes. It is apparent from Fig. 4 that nanoprobes with higher surface coverage of hpDNA displayed higher target-capture rate. The hybridization rates could be quantitatively obtained as the first-ordered differentiation of the curve in Fig. 4. It was found that, in the initial rapid hybridization period upon target addition, the response of NR-hpDNA400 to c-DNA was about 1.4 and 5.3 times faster than that of NRhpDNA200 and NR-hpDNA100, respectively. The fluorescence intensities of NR-hpDNA400 and NR-hpDNA200 reached saturation levels in a similar time period, while a longer time was needed for NR-hpDNA100.

As also shown in Fig. 4, the fluorescence intensity of FAM in the absence of target strands, i.e., background signal, was low but measureable for all three nanoprobes. The background signal of NR-hpDNA400 was relatively higher than that of NRhpDNA200, while the latter was just slightly higher than that of NR-hpDNA100. The saturation fluorescence signal in the presence of targets, on the other hand, was found to have a positive relationship with hpDNA loading. In addition to fluorescence intensity, which is primarily related to the number of open hpDNA, another factor usually used to determine the sensor performance of MB is the quenching efficiency, defined as $\left(1-F_{\text {closed }} / F_{\text {open }}\right) \times 100 \%$, where $F_{\text {closed }}$ and $F_{\text {open }}$ are the fluorescence intensity of the nanoprobe in the absence of target and its stable level in the presence of excess target, respectively. For NR-hpDNA400, NR-hpDNA200, and NR-hpDNA100, the quenching efficiencies were calculated to be $90.8 \%, 93.3 \%$, and $88.1 \%$, respectively, indicating a similar and good quenching effect of all three nanoprobes.

To further evaluate the effectiveness of the NR-hpDNA nanoprobes for nucleic acid detection, the average number of target strands hybridized with hpDNA on each nanoprobe was quantified using the protocol reported by Demers et al. ${ }^{38}$ An excess of TMR-c-DNA ( $3 \mu \mathrm{M}$ as final concentration) was incubated with NR-hpDNA nanoprobes in the hybridization buffer for $24 \mathrm{~h}$ to maximize the hybridizations. The influence of TMR labels on the hybridization (duplex formation) is negligible. ${ }^{48}$ Following a centrifuge process to remove unbound excess TMR-c-DNA, the hybridized TMR-c-DNA were released by denaturing the duplex DNA and separation from the NR-hpDNA nanoprobes. The concentration of dehybridized TMR-c-DNA was deduced from its fluorescence intensity according to a concentration-intensity correlation curve. Table 1 lists the surface coverage of hairpins, surface coverage of hybridized hairpins (with TMR-c-DNA), and hybridization efficiency of three NR-hpDNA nanoprobes. Interestingly, the number of captured target strands increased with increasing surface coverage of hpDNA on GNR, which is consistent with the saturate fluorescence intensity observed in the kinetic studies (Fig. 4). However, the hybridization ratio decreased from $94.44 \%$ to $27.19 \%$ as the hpDNA packing density increased from $0.87 \times 10^{12}$ to $5.54 \times 10^{12} \mathrm{hpDNA} / \mathrm{cm}^{2}$. This indicates that a higher hairpin density results in an increased total target binding, but a relatively lower efficiency in hybridizing hpDNA available on GNR. ${ }^{49,50}$ This is not surprising as previous studies have found that, for both DNA on thin films and nanoparticles, the efficiency of DNA hybridization is governed by both the electrostatic repulsion between neighboring DNA strands and the steric hindrance between tethered DNA probes. ${ }^{38,49,51}$ An upright conformation of oligonucleotide, which is preferred for hpDNA of relatively high surface coverage due to the repulsive force between neighboring oligonucleotides, is favorable for hybridizations. On the other hand, densely packed oligonucleotide monolayers would reduce accessibility of incoming target strands.

Figure 5 depicts the correlation between fluorescence intensity of nanoprobes and the target concentration. As expected, for all nanoprobes, the recovery of the fluorescence signal was positively correlated to the target concentration. Apparent changes in the fluorescence intensities were observed at a target concentration of $1 \mathrm{nM}$. As the concentration of c-DNA increased, the fluorescence intensity increased monotonically until saturated at a stable plateau at relatively high target concentration. These

Table 1 Hybridization efficiency of NR-hpDNA nanoprobes with different probe surface packing densities.

\begin{tabular}{lccr} 
Samples & Surface coverage $\left(\mathrm{hpDNA} / \mathrm{cm}^{2}\right)$ & Hybridized coverage $\left(\mathrm{hpDNA} / \mathrm{cm}^{2}\right)$ & Hybridization efficiency $(\%)$ \\
\hline NR-hpDNA400 & $(5.54 \pm 0.13) \times 10^{12}$ & $(1.51 \pm 0.01) \times 10^{12}$ & 27.19 \\
NR-hpDNA200 & $(2.33 \pm 0.05) \times 10^{12}$ & $(1.21 \pm 0.05) \times 10^{12}$ & 52.08 \\
NR-hpDNA100 & $(0.87 \pm 0.07) \times 10^{12}$ & $(0.83 \pm 0.07) \times 10^{12}$ & 94.44 \\
\hline
\end{tabular}




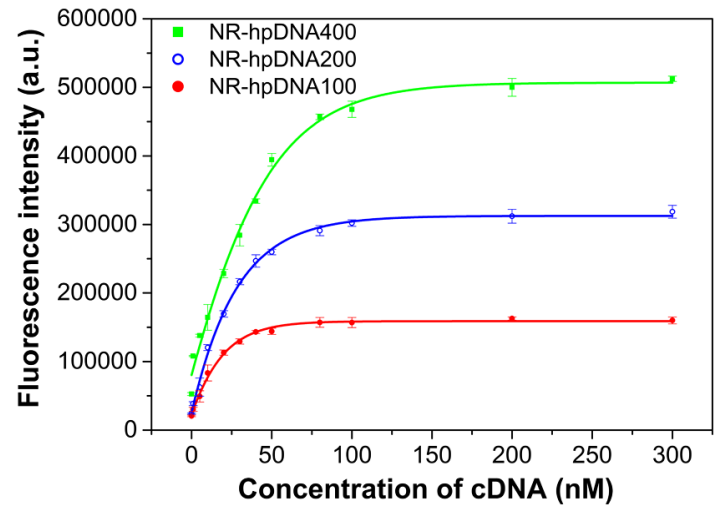

Fig. 5 Dose response of the nanoprobes $(0.22 \mathrm{nM})$ with different surface packing densities of hpDNA. The concentrations of perfectly cDNA were 0, 1, 5, 10, 20, 30, 40, 50, 80, 100, 200, and $300 \mathrm{nM}$. Excitation wavelength: $485 \mathrm{~nm}$; emission peak: $517 \mathrm{~nm}$.

again indicated the opening of hairpin structure upon hybridization. Significantly, nanoprobes of higher hairpin coverage not only showed stronger fluorescence intensity at the same target concentration, but also a higher saturation signal at a larger target saturation concentration. This means that the nanoprobes of higher hairpin coverage had better sensitivity and larger detection range. The limit of detection of probe NRhpDNA400 (LOD $=3.3 \times$ standard deviation of the response/ the slope of the calibration curve up to $50 \mathrm{nM}$ ) was found to be $0.68 \mathrm{nM}$.

Furthermore, time-resolved fluorescence spectroscopy was employed to evaluate the lifetime change of FAM on the nanoprobes before and after being hybridized with targets. For comparison, the fluorescence intensity decay of free hpDNA-FAM was analyzed prior to conjugation to GNR. Two lifetime components were found to present in the free hpDNA-FAM sample, where the long lifetime of $3.92 \mathrm{~ns}$ was the dominant one, accounting for a fractional contribution of $98 \%$. It is worth noting that the fluorescence decay of free hpDNAFAM in "closed" state (without binding to c-DNA) was slightly different from that in "open" state (hybridized with c-DNA) with both long and short lifetimes of free open-state hpDNA-FAM slightly greater than those of hpDNA-FAM in "closed" state, as determined from multiexponential analysis (Table 2). This is probably due to the close proximity of FAM to the guanine in the hairpin conformation. Previous studies found that the fluorescence of FAM could be quenched by guanosine nucleotide due to the photoinduced electron transfer. ${ }^{52-54}$ Indeed, the hpDNA-FAM hybridized with c-DNA showed a 1.7-fold increase in fluorescence intensity with respect to the hpDNAFAM in the "closed" state.

After being assembled on GNRs, the FAM molecules were held to the close proximity of GNR surfaces by the hairpin DNA structure in the absence of targets. Consequently, the fluorescence lifetime of FAM was dramatically shortened due to the fluorescence quenching effect induced by GNR. Multiexponential fitting shows that the FAM in NRhpDNA400 has three lifetime components of 2.87 ns (8\%), $0.85 \mathrm{~ns}(59 \%)$, and $0.35 \mathrm{~ns}(33 \%)$ (Table 2). As noted, the shorter lifetime components of $<1$ ns were dominant in the fluorescence decay. The total average lifetime was calculated to be $0.85 \mathrm{~ns}$, about 4.5 -fold smaller than that of the free closed-state hpDNA-FAM, confirming the quenching effect introduced by GNR. Upon target binding, the fluorescence lifetime of
Table 2 Multiexponential analysis of fluorescence intensity decays.

\begin{tabular}{|c|c|c|c|c|}
\hline Samples & $\bar{\tau}(\mathrm{ns})^{\mathrm{a}}$ & $\tau_{i}(\mathrm{~ns})^{\mathrm{b}}$ & $f_{i}^{\mathrm{a}}$ & $\chi_{R}^{2}$ \\
\hline \multirow[t]{2}{*}{ hpDNA } & 3.87 & $3.92 \pm 0.01$ & 0.98 & \\
\hline & & $0.47 \pm 0.11$ & 0.02 & 1.14 \\
\hline \multirow[t]{2}{*}{ hpDNA + c-DNA } & 3.93 & $4.05 \pm 0.02$ & 0.94 & \\
\hline & & $2.03 \pm 0.07$ & 0.06 & 1.08 \\
\hline \multirow[t]{3}{*}{ NR-hpDNA400 } & 0.85 & $2.87 \pm 0.15$ & 0.08 & \\
\hline & & $0.85 \pm 0.03$ & 0.59 & \\
\hline & & $0.35 \pm 0.05$ & 0.33 & 1.11 \\
\hline \multirow[t]{3}{*}{ NR-hpDNA400 + c-DNA } & 1.40 & $3.93 \pm 0.10$ & 0.23 & \\
\hline & & $1.05 \pm 0.10$ & 0.24 & \\
\hline & & $0.45 \pm 0.03$ & 0.53 & 1.20 \\
\hline \multirow[t]{3}{*}{ NR-hpDNA200 } & 0.83 & $2.64 \pm 0.18$ & 0.11 & \\
\hline & & $0.79 \pm 0.04$ & 0.57 & \\
\hline & & $0.31 \pm 0.06$ & 0.33 & 1.18 \\
\hline \multirow[t]{3}{*}{ NR-hpDNA200 + c-DNA } & 2.71 & $3.96 \pm 0.03$ & 0.62 & \\
\hline & & $1.15 \pm 0.16$ & 0.13 & \\
\hline & & $0.41 \pm 0.05$ & 0.25 & 1.09 \\
\hline \multirow[t]{3}{*}{ NR-hpDNA100 } & 0.84 & $2.10 \pm 0.21$ & 0.10 & \\
\hline & & $0.81 \pm 0.05$ & 0.51 & \\
\hline & & $0.33 \pm 0.05$ & 0.40 & 1.13 \\
\hline \multirow[t]{3}{*}{ NR-hpDNA100 + c-DNA } & 3.02 & $3.95 \pm 0.03$ & 0.72 & \\
\hline & & $1.25 \pm 0.25$ & 0.09 & \\
\hline & & $0.39 \pm 0.05$ & 0.20 & 1.03 \\
\hline
\end{tabular}

aThe fluorescence decay was fitted to three exponentials plus scatter to take into account the scatter effect caused by GNR and the amplitude of scatter was excluded from the data analysis.

${ }^{\mathrm{b}}$ The retrieved lifetimes are presented with three standard deviations as error.

FAM recovered as expected, with three lifetime components of $3.93 \mathrm{~ns}(23 \%), 1.05 \mathrm{~ns}(24 \%)$, and $0.45 \mathrm{~ns}$ (53\%) (Table 2). The average lifetime was $1.40 \mathrm{~ns}$, about a 1.6-fold increase compared to that of the closed-state nanoprobe. All of the lifetime components increased with the longest one approaching that of free open-state hpDNA-FAM. The fractional contribution of the longest lifetime component significantly increased, whereas the fractional contribution of shorter components decreased. The existence of short lifetime components indicated that not all hpDNA opened, in line with previous finding that about $27 \%$ of hpDNA were in open states. The fluorescence lifetime distributions retrieved from MEM are shown in Fig. 6(d). By summing the area under the peaks, the fractional contributions for a continuous lifetime distribution can be determined. The MEM analysis reveals that the lifetime spectrum of NR-hpDNA400 in the absence of c-DNA consists of three peaks located at $3.37 \mathrm{~ns}$ 
(22\%), $0.93 \mathrm{~ns}(52 \%)$, and $0.44 \mathrm{~ns}(22 \%)$ and one extremely small distribution centered at $0.06 \mathrm{~ns}(1 \%)$. Significantly, upon hybridization to c-DNA, the 3.37-ns lifetime component shifted toward a greater value (4.06 ns) with the fractional contribution increasing to $63 \%$, whereas the 0.93 -ns band shifted to $1.18 \mathrm{~ns}$ with the fractional contribution falling to $17 \%$. Meanwhile, the second shortest lifetime peak became relatively sharp and narrow with barycenter at $0.48 \mathrm{~ns}$ and fractional contribution of $20 \%$. In contrast, the contribution from the shortest lifetime peak $(0.04 \mathrm{~ns})$ almost vanished in the fluorescence decay, only accounting for a fractional contribution of $0.37 \%$. The average lifetimes of NR-hpDNA400 before and after hybridizations were calculated to be 1.6 and $2.9 \mathrm{~ns}$, respectively. Due to the complexity of fluorescence decay in the NR-hpDNAFAM system, it is not surprising that there are discrepancies between the fitting results obtained from MEM and multiexponential models. Nevertheless, the MEM analysis is qualitatively consistent with the multiexponential analysis. The kinetics revealed by the fluorescence lifetime measurements are in accordance with the observations obtained by steady-state fluorescence spectroscopy (Fig. 4).

Comparing the closed-state nanoprobes with different hpDNA densities, multiexponential analysis showed similar average lifetimes of $\sim 0.8 \mathrm{~ns}$ (Table 2 ), suggesting a similar hairpin configuration for all three nanoprobes. However, the change of average lifetime upon hybridization was found to be dependent on the hpDNA density. After binding to targets, three lifetime components were found similar for all three nanoprobes (Table 2). However, the corresponding fractional contributions of similar lifetime component were different. As the surface density of hpDNA decreased, the fractional contribution of the longest lifetime component ( $3.95 \mathrm{~ns})$ increased and became dominant in the fluorescence decay, whereas the fractional contributions of the shorter lifetime components decreased. The average lifetimes of NR-hpDNA400, NR-hpDNA200, and NR-hpDNA100 after binding to targets were 1.40, 2.71, and 3.02 ns, respectively. As shown in Figs. 6(e) and 6(f), the lifetime spectra retrieved from MEM clearly demonstrate that the longest lifetime distribution centered at $\sim 4$ ns played an essential role in the decay of the hybridized nanoprobes, whereas the relatively broad lifetime distribution ranging from 0.2 to $1.5 \mathrm{~ns}$ was predominant in the nonhybridized samples. Moreover, the MEM analysis shows that the average lifetimes of NR-hpDNA400, NR-hpDNA200, and NR-hpDNA100 were $~ 1.6$ ns before hybridization, but increased to $2.9,3.7$, and $3.8 \mathrm{~ns}$, respectively, after exposure to an excess of target strands. These were again in agreement with the multiexponential analysis results. The changes in average lifetime revealed by both fitting methods were in line with hybridization efficiency found in Table 1. However, the average lifetime for NR-hpDNA100 after hybridization was still smaller than that of free hybridized DNAs, although $94 \%$ of hpDNA were hybridized as revealed above. This is possibly because not all hybridized hpDNAs fully stretched out from the GNR surface, due to low hpDNA packing
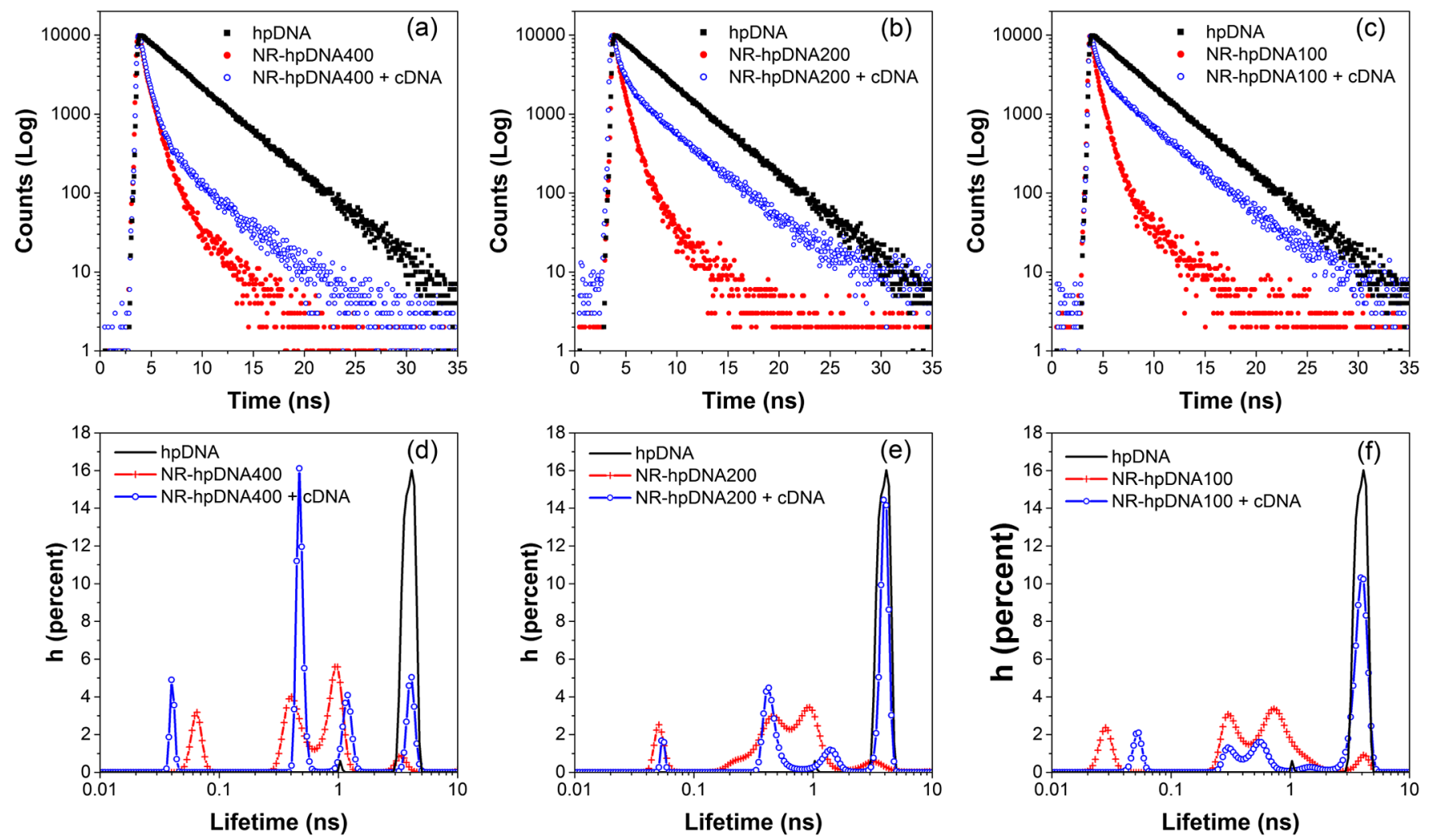

Fig. 6 (Upper panel) fluorescence intensity decay curves of (a) NR-hpDNA400, (b) NR-hpDNA200, and (c) NR-hpDNA100 before and after hybridization ([c-DNA] $=880 \mathrm{nM})$. The fluorescence intensity decay curve of hpDNA was also presented for comparison. Samples were measured in $10 \mathrm{mM}$ phosphate buffer (pH 7.5). (Lower panel) fluorescence lifetime distributions of (d) NR-hpDNA400, (e) NR-hpDNA200, and (f) NR-hpDNA100 before and after hybridization obtained from MEM analysis. The fluorescence lifetime distribution of hpDNA was also included for comparison. Note the logarithmic lifetime axis. 
density and lacking of electrostatic repulsions from the neighbors. This indicates that time-resolved fluorescence spectroscopy is a powerful technique not only for providing information related to hairpin conformational changes, as demonstrated recently, ${ }^{55}$ but also to hybridization ratio of assembled hpDNAs.

\section{Conclusions}

In summary, a GNR-based nanoprobe with potential for mRNA detection was developed by functionalizing GNR with fluorophore-labeled hairpin oligonucleotides. This nanoprobe was found to be sensitive to a complementary oligonucleotide as indicated by significant changes in fluorescence intensity and lifetime. Tuneable loading of hpDNA on GNR was achieved by varying the molar ratio of hpDNA to GNR during the functionalization process. It was found that the nanoprobe of higher hairpin coverage showed better performance in terms of sensitivity and detection range from the steady-state fluorescence spectroscopy measurement. It was also found that nanoprobes of the highest hairpin density captured the largest number of target strands, but had a relatively low hybridization ratio. Analysis by time-resolved fluorescence lifetime spectroscopy revealed significant lifetime changes of the fluorophore after hpDNAs hybridized with targets. It demonstrated that timeresolved fluorescence spectroscopy can be a powerful tool for providing insight on the hybridization kinetics of the probe as well as the quenching effect of GNR. We expect that this kind of GNR-based nanoprobe holds promise for mRNA detection and subcellular imaging with the concomitant potential for a wide range of disease related biomarker RNA analyses, including cancer diagnosis and prognosis.

\section{Acknowledgments}

We thank Dr J. Sutter, P. Yip, and W. Li for assistance during the experimental work. This work was funded by the BBSRC (BB/K013416/1). G.W. acknowledges financial support from the China Scholarship Council (CSC).

\section{References}

1. C. E. Holt and S. L. Bullock, "Subcellular mRNA localization in animal cells and why it matters," Science 326, 1212-1216 (2009).

2. C. Eliscovich et al., "mRNA on the move: the road to its biological destiny," J. Biol. Chem. 288, 20361-20368 (2013).

3. R. W. Dirks, C. Molenaar, and H. J. Tanke, "Methods for visualizing RNA processing and transport pathways in living cells," Histochem. Cell Biol. 115, 3-11 (2001).

4. S. Tyagi and F. R. Kramer, "Molecular beacons: probes that fluoresce upon hybridization," Nat. Biotechnol. 14, 303-308 (1996).

5. S. Tyagi, D. P. Bratu, and F. R. Kramer, "Multicolor molecular beacons for allele discrimination," Nat. Biotechnol. 16, 49-53 (1998).

6. T. Matsuo, "In situ visualization of messenger RNA for basic fibroblast growth factor in living cells," Biochim. Biophys. Acta 1379, 178-184 (1998).

7. D. L. Sokol et al., "Real time detection of DNA.RNA hybridization in living cells," Proc. Natl. Acad. Sci. U. S. A. 95, 11538-11543 (1998).

8. N. Nitin et al., "Peptide-linked molecular beacons for efficient delivery and rapid mRNA detection in living cells," Nucleic Acids Res. 32, e58 (2004).

9. C. D. Medley et al., "Simultaneous monitoring of the expression of multiple genes inside of single breast carcinoma cells," Anal. Chem. 77, 4713-4718 (2005).

10. B. Dubertret, M. Calame, and A. J. Libchaber, "Single-mismatch detection using gold-quenched fluorescent oligonucleotides," Nat. Biotechnol. 19, 365-370 (2001).
11. Y. Zhang, D. J. S. Birch, and Y. Chen, "Two-photon excited surface plasmon enhanced energy transfer between dapi and gold nanoparticles: opportunities in intra-cellular imaging and sensing," Appl. Phys. Lett. 99, 103701 (2011).

12. C. Racknor et al., "Energy transfer between a biological labelling dye and gold nanorods," Methods Appl. Fluoresc. 2, 015002 (2014).

13. P. Gu, D. J. S. Birch, and Y. Chen, "Dye-doped polystyrene-coated gold nanorods: towards wavelength tuneable SPASER," Methods Appl. Fluoresc. 2, 024004 (2014).

14. S. Mayilo et al., "Long-range fluorescence quenching by gold nanoparticles in a sandwich immunoassay for cardiac troponin T," Nano Lett. 9, 4558-4563 (2009).

15. N. L. Rosi et al., "Oligonucleotide-modified gold nanoparticles for intracellular gene regulation," Science 312, 1027-1030 (2006).

16. D. S. Seferos et al., "Nano-flares: probes for transfection and mRNA detection in living cells," J. Am. Chem. Soc. 129, 15477-15479 (2007).

17. A. E. Prigodich et al., "Nano-Flares for mRNA regulation and detection," ACS Nano 3, 2147-2152 (2009).

18. S. Song et al., "Gold-nanoparticle-based multicolor nanobeacons for sequence-specific DNA analysis," Angew. Chemie Int. Ed. 48, 86708674 (2009).

19. W. Pan et al., "Dual-targeted nanocarrier based on cell surface receptor and intracellular mRNA: an effective strategy for cancer cell imaging and therapy," Anal. Chem. 85, 6930-6935 (2013).

20. W. Pan et al., "Multiplexed detection and imaging of intracellular mRNAs using a four-color nanoprobe," Anal. Chem. 85, 10581-10588 (2013).

21. A. Jayagopal et al., "Hairpin DNA-functionalized gold colloids for the imaging of mRNA in live cells," J. Am. Chem. Soc. 132, 9789-9796 (2010).

22. S. R. Harry et al., "Hairpin DNA coated gold nanoparticles as intracellular mRNA probes for the detection of tyrosinase gene expression in melanoma cells," Chem. Commun. 46, 5557-5559 (2010).

23. C. J. Murphy et al., "Anisotropic metal nanoparticles: synthesis, assembly, and optical applications," J. Phys. Chem. B 109, 1385713870 (2005).

24. Y. Chen, J. A. Preece, and R. E. Palmer, "Processing and characterization of gold nanoparticles for use in plasmon probe spectroscopy and microscopy of biosystems," Ann. N. Y. Acad. Sci. 1130, 201-206 (2008).

25. R. Weissleder, "A clearer vision for in vivo imaging," Nat. Biotechnol. 19, 316-317 (2001).

26. G. Boyd, Z. Yu, and Y. Shen, "Photoinduced luminescence from the noble metals and its enhancement on roughened surfaces," Phys. Rev. B 33, 7923-7936 (1986).

27. H. Wang et al., "In vitro and in vivo two-photon luminescence imaging of single gold nanorods," Proc. Natl. Acad. Sci. U. S. A. 102, 1575215756 (2005).

28. Y. Chen et al., "Creation and luminescence of size-selected gold nanorods," Nanoscale 4, 5017-5022 (2012).

29. Y. Zhang et al., "Gold nanorods for fluorescence lifetime imaging in biology," J. Biomed. Opt. 15, 020504 (2010).

30. A. Agarwal et al., "Targeted gold nanorod contrast agent for prostate cancer detection by photoacoustic imaging," J. Appl. Phys. 102, 064701 (2007).

31. C.-C. Chen et al., "DNA-gold nanorod conjugates for remote control of localized gene expression by near infrared irradiation," J. Am. Chem. Soc. 128, 3709-3715 (2006).

32. X. Huang et al., "Cancer cell imaging and photothermal therapy in the near-infrared region by using gold nanorods," J. Am. Chem. Soc. 128, 2115-2120 (2006).

33. Y. Zhang et al., "Surface plasmon enhanced energy transfer between gold nanorods and fluorophores: application to endocytosis study and RNA detection," Faraday Discuss. 178, 383-394 (2015).

34. B. Nikoobakht and M. A. El-Sayed, "Preparation and growth mechanism of gold nanorods (NRs) using seed-mediated growth method," Chem. Mater. 15, 1957-1962 (2003).

35. T. K. Sau and C. J. Murphy, "Seeded high yield synthesis of short Au nanorods in aqueous solution," Langmuir 20, 6414-6420 (2004).

36. A. Wijaya and K. Hamad-Schifferli, "Ligand customization and DNA functionalization of gold nanorods via round-trip phase transfer ligand exchange," Langmuir 24, 9966-9969 (2008). 
37. C. J. Orendorff and C. J. Murphy, "Quantitation of metal content in the silver-assisted growth of gold nanorods," J. Phys. Chem. B 110, 3990-3994 (2006).

38. L. M. Demers et al., "A fluorescence-based method for determining the surface coverage and hybridization efficiency of thiol-capped oligonucleotides bound to gold thin films and nanoparticles," Anal. Chem. 72, 5535-5541 (2000).

39. K. A. Brown, S. Park, and K. Hamad-Schifferli, "Nucleotide-surface interactions in DNA-modified Au-nanoparticle conjugates: sequence effects on reactivity and hybridization," J. Phys. Chem. C 112, 7517-7521 (2008).

40. S. J. Hurst, A. K. R. Lytton-Jean, and C. A. Mirkin, "Maximizing DNA loading on a range of gold nanoparticle sizes," Anal. Chem. 78, 8313-8318 (2006).

41. A. Wijaya et al., "Selective release of multiple DNA oligonucleotides from gold nanorods," ACS Nano 3, 80-86 (2009).

42. J. Brochon, "Maximum entropy method of data analysis in timeresolved spectroscopy," Methods Enzymol. 240, 262-311 (1994).

43. A. M. Alkilany et al., "Cellular uptake and cytotoxicity of gold nanorods: molecular origin of cytotoxicity and surface effects," Small 5, 701-708 (2009).

44. Y. Zhang et al., "Effect of size, shape, and surface modification on cytotoxicity of gold nanoparticles to human HEp-2 and canine MDCK cells," J. Nanomater. 2012, 1-7 (2012).

45. V. Sharma, K. Park, and M. Srinivasarao, "Shape separation of gold nanorods using centrifugation," Proc. Natl. Acad. Sci. U. S. A. 106, 4981-4985 (2009).

46. H. D. Hill et al., "The role radius of curvature plays in thiolated oligonucleotide loading on gold nanoparticles," ACS Nano 3, 418-424 (2009).
47. S. Tyagi et al., "Molecular beacons: hybridization probes for detection of nucleic acids in homogeneous solutions," in Nonradioactive Analysis of Biomolecules, C. Kessler, Ed., pp. 606-616 Springer (2000).

48. B. G. Moreira et al., "Effects of fluorescent dyes, quenchers, and dangling ends on DNA duplex stability," Biochem. Biophys. Res. Commun. 327, 473-484 (2005).

49. A. W. Peterson, R. J. Heaton, and R. M. Georgiadis, "The effect of surface probe density on DNA hybridization," Nucleic Acids Res. 29, 5163-5168 (2001).

50. K. B. Cederquist and C. D. Keating, "Hybridization efficiency of molecular beacons bound to gold nanowires: effect of surface coverage and target length," Langmuir 26, 18273-18280 (2010).

51. R. Levicky et al., "Using self-assembly to control the structure of DNA monolayers on gold: a neutron reflectivity study," J. Am. Chem. Soc. 120, 9787-9792 (1998).

52. A. O. Crockett and C. T. Wittwer, "Fluorescein-labeled oligonucleotides for real-time PCR: using the inherent quenching of deoxyguanosine nucleotides," Anal. Biochem. 290, 89-97 (2001).

53. J. R. Unruh et al., "Fluorescence properties of fluorescein, tetramethylrhodamine and texas red linked to a DNA aptamer," Photochem. Photobiol. 81, 682-690 (2005).

54. T. Heinlein et al., "Photoinduced electron transfer between fluorescent dyes and guanosine residues in DNA-hairpins," J. Phys. Chem. B 107, 7957-7964 (2003).

55. G. Wei et al., "Revealing the photophysics of gold-nanobeacons via time-resolved fluorescence spectroscopy," Opt. Lett. 40, 5738-5741 (2015).

Biographies for the authors are not available. 